\title{
ON THE TEMPERATURE DEPENDENCE OF ELECTRICAL RESISTIVITY IN CONCENTRATED DISORDERED TRANSITION BINARY ALLOYS $(*)$
}

\author{
F. BROUERS
}

\author{
Laboratoire de Physique des Solides $\left({ }^{* *}\right)$, Université Paris-Sud, Centre d'Orsay \\ 91405 Orsay, France \\ and
}

M. BRAUWERS $(* * *)$

Institut de Physique, Université de Liège, Sart-Tilman, 4000 Liège, Belgique

(Reçu le 17 juin 1974, révisé le 5 novembre 1974)

\begin{abstract}
Résumé. - En utilisant un modèle à deux bandes et la théorie du CPA de la conductibilité, on calcule l'effet de la modification de densité d'état induite par les phonons sur la dépendance en $T$ de la résistivité électrique dans les alliages concentrés de métaux de transition. On montre que cet effet n'est pas négligeable et que, dans certains cas, il peut produire une valeur négative des coefficients $\mathrm{d} \rho / \mathrm{d} T$ comme cela est observé dans certains de ces alliages.

Abstract. - Using a two (s-d) band model and the CPA theory of conductivity, we calculate the effect of the phonon induced modification of the density of states on the $T$-dependence of the electrical resistivity in concentrated transition metal alloys. It is shown that this effect is not at all negligible and in some cases can yield a negative $T$-coefficient $\mathrm{d} \rho / \mathrm{d} T$ as observed in some of these alloys.
\end{abstract}

1. Introduction. - As is now well known, a number of concentrated disordered transition metal alloys exhibit a temperature coefficient of resistance $a=\mathrm{d} \rho / \mathrm{d} T$ which is much lower than would be expected from Matthiessen's rule [1]. Metallic systems with a high $T$-independent resistivity are of great interest for producing $T$-independent high-quality resistors. Among these alloys, one can mention highly concentrated disordered alloys of $\mathrm{NiCr}$ [2], $\mathrm{CuNi}$ [3], FeCrAl [4] and AgPd [5]. In these systems, the low $a$ coefficient is not observed in the pure metals but only when the alloys are rather concentrated. Negative $a$ 's are observed in many alloys, generally in a restricted range of temperature and close to some magnetic or structural transitions. The best known example is the negative $a$ observed in some $\mathrm{CuNi}$ alloys just above the ferromagnetic Curie point. In these alloys, the general belief is that this anomalous behaviour is related to magnetic or chemical short-range order which decreases with $T$. In some alloys however like TiV [5], a negative $a$ has been observed over a large range of $T$.

Another interesting point emphasized by Mooij [1]

(*) Partially supported by ESIS Programme.

(**) Laboratoire associé au C. N. R. S.

$(* * *)$ Aspirant au F. N. R. S., Brussels. is the correlation between $a$ and the magnitude of the resistivity. If one excludes data on alloys where $a$ may be affected by magnetic or structural transition, it appears that no negative $a$ is observed below $100 \mu \Omega$ while hardly any positive $a$ is found above $150 \mu \Omega \mathrm{cm}$.

Very recently, Ahmad and Greig [7] have discussed the occurrence in $\mathrm{Pd}_{60} \mathrm{Ag}_{40}$ of a decrease of the electrical resistivity with increasing $T$. in the range 300$700 \mathrm{~K}$. These authors argue that in this alloy the decrease of the resistivity cannot be attributed to the dispersion of giant moment clouds as in $\mathrm{NiCu}$ since the latter effect occurs in metals which are nearly ferromagnetic and this is certainly not the case in $\operatorname{Pd}_{60} \mathrm{Ag}_{40}$. In the case of PdAg alloys, the traditional explanation [8] is that such $T$-dependence is due to the Fermi smearing of the effective density of states $g_{\mathrm{d}}(E) f_{\mathrm{FD}}(E)$. Coles and Taylor [9] emphasized that this effect would yield a $T$-modulation of the residual resistivity due to s-d scattering in PdAg alloys and the observed negative $\mathrm{d} \rho / \mathrm{d} T$.

The purpose of this letter is to consider the effect of the first factor of the effective density of states on the $T$-dependence of the resistivity i. e. the variation of $g_{\mathrm{d}}(E)$ with $T$ due to its smearing by the electron-phonon interaction.

Recent calculations [10] of the pressure dependence 
of the electronic structure and Fermi surface of Pd indicate that a change of $2 \%$ in the lattice constant changes the density of states at the Fermi-energy by about $10 \%$. This is the order of magnitude of the effect of vibrations of the lattice at room temperature.

The theory of concentrated alloys is still in its early development and we are not yet able to provide any quantitative first principle description of the transport properties of transition metal alloys. Moreover there are no theoretical grounds for believing that there is a common explanation for the anomalous behaviour of the resistivity with respect to temperature because of the complicated interplay between configuration, thermal and magnetic disorder. However, one thing we know for certain is that we cannot be content with a rigid band description for concentrated alloys such as PdAg or $\mathrm{NiCu}$. X-ray photoelectron spectra $[11,12]$ indicate clearly that the density of states of these alloys presents a double peak structure corresponding to the peaks of the two component alloy subbands whose relative importance varies with concentration. Model calculations have been used within the framework of the coherent potential approximation (CPA) to account for this behaviour in transition metal alloys [13]. One can use these recent developments of the alloy theory to make predictions concerning the trend of the variation of the transport coefficients with the parameters characterizing the alloy : concentration and electronic and vibrational scattering parameters.

A first attempt to use CPA theory to deal with the problem we are interested in here, the $T$-dependence of the electrical resistivity, was presented by Chen et al. [14]. This is a model calculation the approximations of which are well defined :

a) The complicated band-structure of the transition metals forming the alloy is described by a tight-binding structureless semi-elliptic band having the same bandwidth for both metals ; one uses a sphericaly symmetric velocity function corresponding to that density of states.

b) One neglects the influence of the neighbourhood configuration on the atomic potential and therefore any clustering phenomenon. The density of states and the conductivity are calculated in the framework of the single-site CPA.

c) The electron-phonon interaction is approximated by a site-diagonal local interaction. The thermal average is calculated assuming that the phonon spectrum reduces to two frequencies $\omega_{A}$ and $\omega_{B}$ characteristic of the pure metals forming the alloy. This gives rise to Gaussian fluctuations of the atomic potentials.

The mean squares of the distributions are linear in $T$ at high temperature and in that limit can be related to the parameters of the metal using deformation potential theory.

From their theoretical and numerical investigation Chen et al. concluded that thermal disorder smears and broadens the electronic density of states in the alloy and that for large values of the scattering parameter, the resistivity may increase, decrease or remain constant with $T$ depending on the location of the Fermi energy. They found that a decrease of the resistivity can occur when the Fermi level lies in the inter-band region in a region of lower density of states. The physical explanation is that in these regions, the effect of high scattering is maximum and electrons are strongly localized. The effect of thermal fluctuations is to assist the motion of these quasi-localized electrons and can in some cases give rise to a decrease of the resistivity with $T$.

However, these results contradict an essential conclusion of Mooij's systematic analysis of experimental data: negative $a$ are correlated with large values of the resistivity which in transition metals and alloys correspond to a high d-density of states.

In this paper, we show that when it is applied to a two (s-d) band model the theory of Chen et al. yields numerical results which are qualitatively in agreement with the conclusions one can draw from Mooij's [1] compilation of transition metal alloy resistivities.

2. Model. - For the discussion of the $T$-dependence of the resistivity of a concentrated binary disordered alloy formed of two transition metals $A_{x} B_{1-x}$, we use a two-band model introduced by Levin and Ehrenreich [15] generalized by Brouers and Vedyayev [16, 17] and then by Gelatt and Ehrenreich [18]. To this Hamiltonian we add the CWS site-diagonal interaction coupling phonons to the more localized d-electrons. The model Hamiltonian which describes a s-band, a d-band, the s-d hybridization and the phonon-delectron coupling reads in a mixed Bloch-Wannier representation

$$
\begin{aligned}
& \mathcal{H}_{\text {alloy }}=\sum_{n} \varepsilon_{n}^{\mathrm{s}}(x)\left|n_{\mathrm{s}}><n_{\mathrm{s}}\right|+\sum_{k} \varepsilon_{\mathrm{s}}(k)\left|k_{\mathrm{s}}><k_{\mathrm{s}}\right|+ \\
& +\sum_{n} \varepsilon_{n}^{\mathrm{d}}(x)\left|n_{\mathrm{d}}><n_{\mathrm{d}}\right|+\sum_{k} \varepsilon_{\mathrm{d}}(k)\left|k_{\mathrm{d}}><k_{\mathrm{d}}\right| \\
& +\sum_{n}\left(\gamma_{n}(x)\left|n_{\mathrm{d}}><n_{\mathrm{s}}\right|+\text { c. c. }\right)+\sum_{n} \theta_{n}\left|n_{\mathrm{d}}><n_{\mathrm{d}}\right|
\end{aligned}
$$

Here

$$
\left|n_{\mathrm{s}(\mathrm{d})}\right\rangle=(1 / \sqrt{N}) \sum_{k} \mathrm{e}^{\mathrm{i} k R_{n}}\left|k_{\mathrm{s}(\mathrm{d})}\right\rangle
$$

corresponds to $\mathrm{s}(\mathrm{d})$ electrons located on site $n$. We assume that only diagonal quantities are random (a generalization of (1) in order to include off-diagonal disorder is considered in Brouers and Van der Rest [19]) : the energies $\varepsilon_{n}^{\mathrm{s}}, \varepsilon_{n}^{\mathrm{d}}$, the s-d electron coupling constant $\gamma_{n}$ and the phonon-d electron coupling constant $\theta_{n}^{\mathrm{d}}$ can take two values characteristic of A or B metal according to the kind of atom occupying the site $n$. The concentration dependence of energies in (1) is the result of a charge transfer mechanism which can be calculated self-consistently $[17,18]$. The solu- 
tion of (1) is somewhat simplified if one assumes that $\mathrm{s}$ and $\mathrm{d}$ dispersion relations are proportional :

$$
\varepsilon_{\mathrm{d}}(k)=D \varepsilon_{\mathrm{s}}(k) \text {. }
$$

The CPA can be applied to Hamiltonian (1). At $T=0$, one determines self-consistently an effective medium by replacing in the CPA one band theory [20] scalar quantities by $2 \times 2$ matrices. The effective Hamiltonian is defined by

$$
\begin{gathered}
\quad\left\langle G>_{\mathrm{c}}=\left(z-\mathcal{H}_{\mathrm{eff}}\right)^{-1} \equiv\right. \\
\equiv\left[\begin{array}{ll}
z-\varepsilon_{\mathrm{s}}(k)-\Sigma_{\mathrm{ss}}(z) & -\Sigma_{\mathrm{sd}}(z) \\
-\Sigma_{\mathrm{sd}}(z) & z-D \varepsilon_{\mathrm{s}}(k)-\Sigma_{\mathrm{dd}}(z)
\end{array}\right]^{-1}
\end{gathered}
$$

and $\Sigma$ is determined by the $2 \times 2$ matrix self-consistent equation :

$$
\left\langle\frac{\varepsilon_{n}-\Sigma}{1-\left(\varepsilon_{n}-\Sigma\right) F}\right\rangle_{\mathrm{c}}=0
$$

$F$ is the site diagonal configuration averaged Green's function : $\left\langle 0\left|\langle G\rangle_{\mathrm{c}}\right| 0\right\rangle$. It has been shown [13], [21], that the application of CPA to (1) yields much better results than in the one-band model. The reason is that one never reaches a true split band regime when the difference between d-potentials is smaller than the s-bandwidth.

Once $\langle G\rangle_{\mathrm{c}}$ is known, one can calculate the CPA conductivity [22] which in this model may be written as the sum of three contributions [16] :

$$
\sigma=\sigma_{\mathrm{ss}}+2 \sigma_{\mathrm{sd}}+\sigma_{\mathrm{dd}}
$$

Brouers and Vedyayev [13] used this model to describe and explain qualitatively some observed deviations from the Nordheim rule for the residual resistivity in transition metal based alloys. The formalism was then extended to treat ferromagnetic alloys [23] to account for the concentration dependence of the anisotropy of resistance in alloys like $\mathrm{NiCu}$. Finally the frequency dependence of the resistivity in noble metal alloys was considered by Brouers et al. [24] and it was shown that the two-band model can explain the observed $\omega^{2}$ dependence of the inverse relaxation time in $\mathrm{AuAg}$ alloys. In this letter we examine the $T$-dependence of the resistivity by calculating the effect of the CWS electronphonon coupling on the resistivity. The results of the calculation are in qualitative agreement with the observations of Mooij [1] and Ahmad and Greig [7].

\section{Temperature dependence of electrical resistivity.} - In the framework of the Born-Oppenheimer adiabatic approximation, following CWS, we decouple the average over all possible positions of the ions in a given configuration and over all possible configurations to get the macroscopic expectation value of the density of states and the electrical conductivity. We follow the approximations $\mathrm{a}, \mathrm{b}$ and $\mathrm{c}$ given in the Introduction.

The configuration average is worked out in the CPA described in section 2 and the thermal average \langle\rangle$_{t}$ is a phonon average over $\theta_{n}^{\mathbf{d}}$ leading to a Gaussian distribution of energy levels. To focus on the essential features of the $s-d$ model we shall consider here the case where only the diagonal quantities $\varepsilon_{n}^{\mathbf{d}}$ and $\theta_{n}^{\mathbf{d}}$ are random. We have therefore to solve the self-consistent equation generalizing (4) for $T \neq 0 \mathrm{~K}$

$$
\begin{aligned}
& \left\langle\left\langle\frac{\varepsilon_{n}^{\mathrm{d}}+\theta_{n}^{\mathrm{d}}-\Sigma_{\mathrm{d}}}{1-\left(\varepsilon_{n}^{\mathrm{d}}-\theta_{n}^{\mathrm{d}}-\Sigma_{\mathrm{d}}\right) F_{\mathrm{dd}}}\right\rangle_{\mathrm{c}}\right\rangle_{\mathrm{t}}= \\
& =\int_{-\infty}^{+\infty} \mathrm{d} \eta\left\langle\left(2 \pi \alpha_{n}\right)^{-1} \times\right. \\
& \left.\times \mathrm{e}^{-\eta^{2} / 2 \alpha_{n}}\left(\frac{\varepsilon_{n}^{\mathrm{d}}+\eta-\Sigma_{\mathrm{d}}}{1-\left(\varepsilon_{n}^{\mathrm{d}}+\eta-\Sigma_{\mathrm{d}}\right) F_{\mathrm{dd}}}\right)\right\rangle_{\mathrm{c}}=0
\end{aligned}
$$

where $\alpha_{n}=|v(n)|^{2} \operatorname{coth}\left(\frac{1}{2} \beta \hbar \omega_{n}\right)$ with $\beta=(k T)^{-1}$. can take two values $\alpha_{A}\left(\omega_{A}\right)$ and $\alpha_{B}\left(\omega_{B}\right)$ and is proportional to the Debye-Waller factor times the square of the electron-phonon coupling constant. Once $\Sigma_{\mathrm{d}}(T)$ is calculated, we can determine the density of states

$$
g(E, T)=g_{\mathrm{s}}(E, T)+g_{\mathrm{d}}(E, T)
$$

the Fermi energy, defined by

$$
\int^{E_{\mathrm{F}}(T)} g\left(E, T, \mathrm{~d} E=x n_{\mathrm{A}}+(1-x) n_{\mathrm{B}}\right.
$$

where $n_{\mathrm{A}}$ and $n_{\mathrm{B}}$ are the numbers of electrons per atom for pure A and B metals and the conductivity $\sigma(T)$ using eq. (59) of reference [16]. In the calculations we have neglected the smearing of the Fermi function. Some representative numerical results are shown in figures 1,2 and 3 . We consider the electrons of a $t_{2 g}$

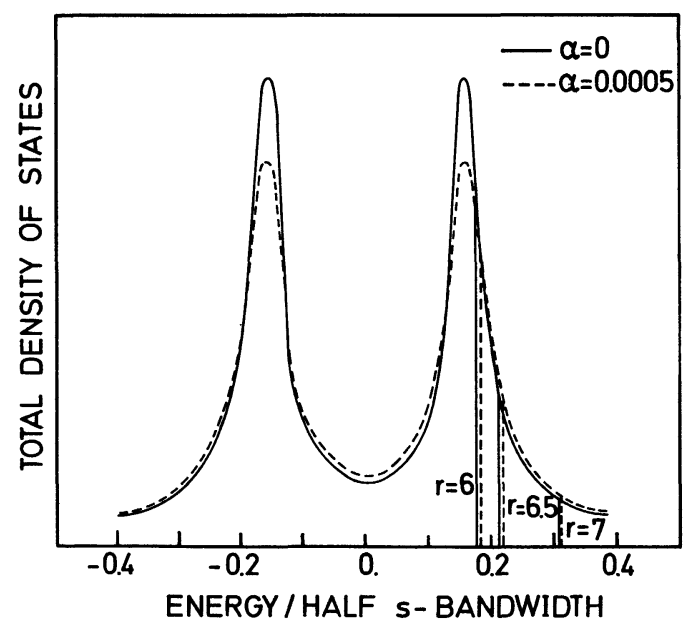

Fig. 1. - Total density of states for two values of $\alpha$. The parameters are $\delta=\varepsilon_{\mathrm{A}}^{\mathrm{d}}-\varepsilon_{\mathrm{B}}^{\mathrm{d}}=2.5 \mathrm{eV}, \gamma=1 \mathrm{eV}, W_{\mathrm{s}}=7.2 \mathrm{eV}$ and $x=0.5$.

$\mathrm{d}$ sub-band whose maximum occupancy is six, coupled to an s-band and we simulate the upper-peak of the b. c. c. d-density of states using the following parameters : an hybridization constant $\gamma=1 \mathrm{eV}$, the coefficient $D$ of eq. (2) $=0.055$. The half s-bandwidth, which 


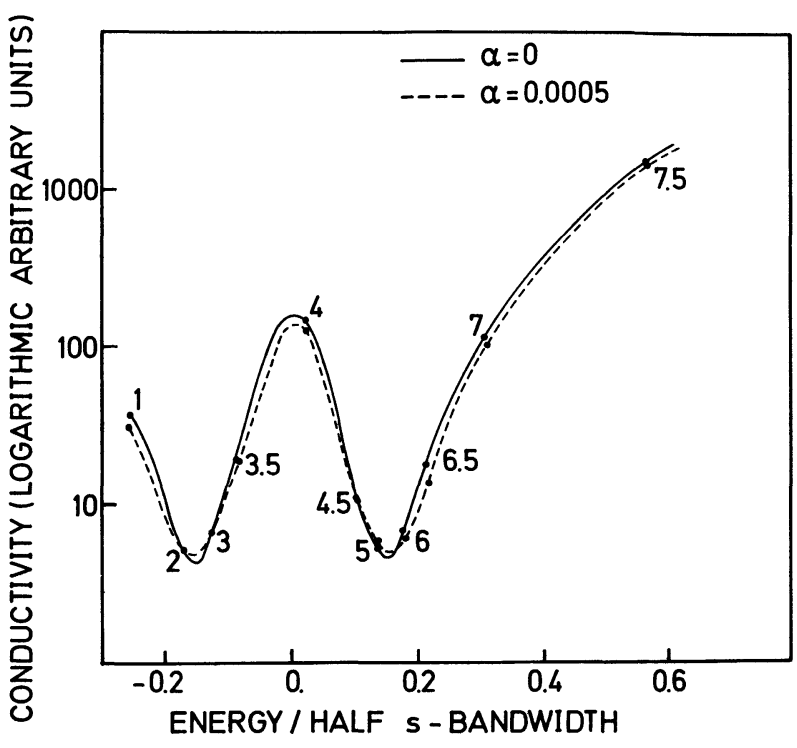

FIG. 2. - Total conductivity for a continuous variation of $E_{\mathrm{F}}$. The figures correspond to the filling of the band.

is the energy unit $W_{\mathrm{s}}=7.2 \mathrm{eV}$. The alloy scattering parameter is $\delta=\varepsilon_{\mathrm{A}}^{\mathrm{d}}-\varepsilon_{\mathrm{B}}^{\mathrm{d}}=2.25 \mathrm{eV}$ and the position of the common s-band is such that its buttom is $5 \mathrm{eV}$ lower than the zero of energies situated midway between $\varepsilon_{\mathrm{A}}^{\mathrm{d}}$ and $\varepsilon_{\mathrm{B}}^{\mathrm{d}}$. The concentration is $50 \%$. Moreover we assume for simplicity that $\omega_{\mathrm{A}}^{\mathrm{d}}=\omega_{\mathrm{B}}^{\mathrm{d}}$ and $\alpha$ is the $T$-dependent parameter proportional to $T$ in the high $T$ limit. An estimate of $\alpha$ for d-electrons is given by Chen et al. [14]: $\alpha \simeq 0.003 W_{\mathrm{d}}^{2} T / T_{\mathrm{m}}$ where $T_{\mathrm{m}}$ is the melting point of pure metals. In our model the effective $\mathrm{d}$-bandwidth is $\sim 0.2$. Figure 1 represents the alloy density of states for $T=0(\alpha=0)$ and for $\alpha=0.0005$. Figure 2 shows the total conductivity for $\alpha=0$ and $\alpha=0.0005$. The two minima correspond to the peaks in the d-density of states. (The numbers on the figure

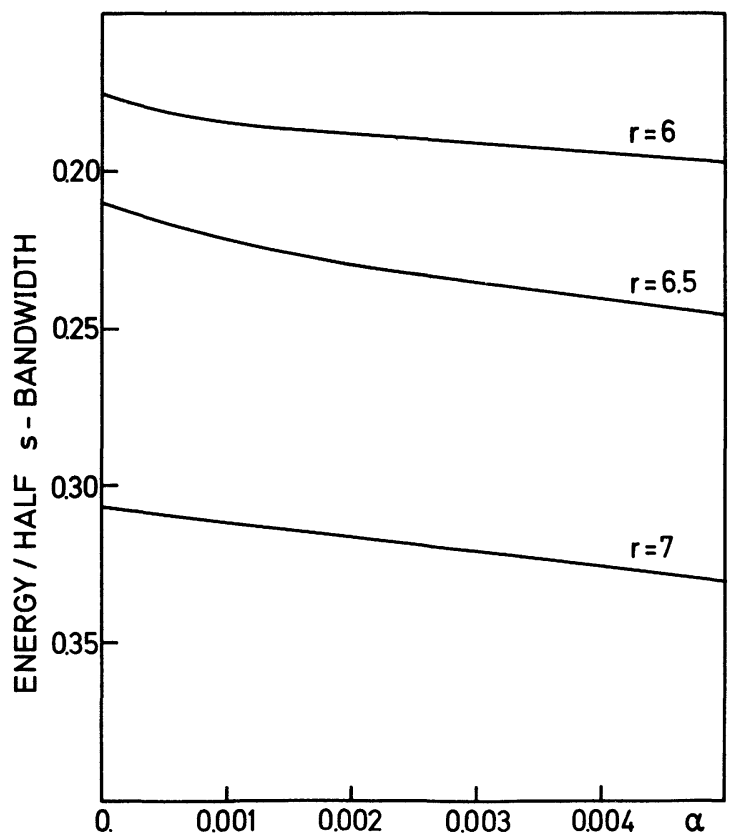

FIG. 3. - Variation of $E_{F}$ with $\alpha$ for three different fillings. correspond to the degree of filling of the band.) Figure 3 exhibits the variation of the Fermi level $E_{\mathrm{F}}$ with $T$ for three characteristic band filling levels and figure 4 which shows the conductivity up to $\alpha=0.0015$ allows us to formulate the conclusions of our calculations.

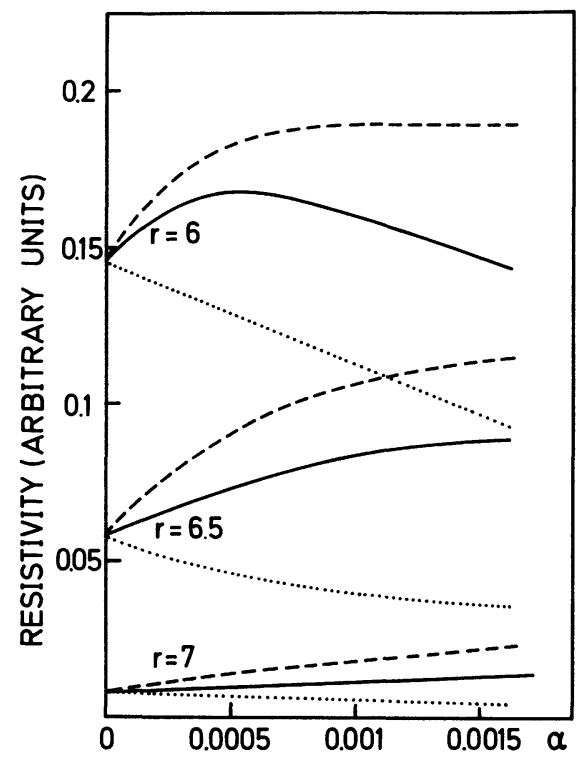

FIG. 4. - Variation of the total resistivity (full time); of the total resistivity supposing a $E_{\mathrm{F}}$ fixed at its $\alpha(T)=0$ value (broken line) ; and of the residual resistivity as $E_{F}$ changes with $\alpha$ (dotted line).

The three chosen band filling levels correspond to three different positions of $E_{\mathrm{F}}$ : for $r=6$ it is located not far from the maximum of a d-peak, for $r=6.5$, $E_{\mathrm{F}}$ is situated outside the d-peak while for $r=7, E_{\mathrm{F}}$ is outside the d-band, though there is still a small s-d coupling. Figure 4 represents the resistivity versus $\alpha$ for these three filling levels. The full lines are the calculated alloy resistivities ; the broken line represents the resistivity supposing $E_{\mathrm{F}}$ fixed at its $T=0$ value; the dotted line shows the variation of the residual resistivity as $E_{\mathrm{F}}$ changes with $\alpha$ (or $T$ ).

Conclusions. - The curves of figure 4 illustrate three different possible regimes : when $E_{\mathrm{F}}$ lies inside the d-band $(r=6,6.5)$, the modification of $\mathrm{s}$ and densities of states, together with the smearing of the d-selfenergy with increasing $\alpha(T)$, tends to yield for high $T$ an almost $T$-independent resistivity. Besides this, the variation of $E_{\mathrm{F}}$ with $T$ (see Fig. 3) gives rise to a decrease of the s-d coupling and therefore of the residual contribution to the resistivity and when this contribution is large enough it can produce a negative $a$-coefficient $(r=6)$. When $E_{\mathrm{F}}$ is outside the d-band $(r=7)$ one finds a behaviour typical of noble metal alloys.

In this letter, we have reported the general trend of results obtained in the two band alloy model : a more detailed analysis will be published elsewhere [25]. We 
did not make any quantitative comparison with experiment since we have neglected among other things the pure s-scattering whose variation with energy and $T$ is much smaller than that of d-scattering but which also contributes to the absolute value of $\rho$.

From the partial results shown here, one can however conclude that the s-d model (1), which has been used to explain qualitatively other independent transport properties of transition metal alloys, accounts for the general behaviour of $\rho(T)$ and $a=\mathrm{d} \rho / \mathrm{d} T$. It explains the correlation observed by Mooij [1] between the values of $\rho$ and $a$ and the fact that a negative $a$ appears only in highly concentrated and high resistivity transition metal alloys.

The transition-noble metal alloys $\mathrm{NiCu}$ and $\mathrm{PdAg}$ have a maximum resistivity smaller than most of the other alloys exhibiting a negative $a$. As a matter of fact the position of the Fermi level corresponds to the case $r=6.5$ and in the present model the effect of electron-phonon interaction, though important, is not sufficient to explain a negative $a$ for these two alloys. This is not surprising since other mechanisms, Fermi function smearing [7-9] in $\mathrm{NiCu}$ and $\mathrm{AgPd}$, and cluster effects [26] in NiCu contribute to the $T$-dependence of the resistivity. To interpret experimental data, one has to consider and analyze every contribution. In this paper, we have shown that the effect of $T$ on the electronic resistivity due to the electron-phonon interaction can be one of the significant contributions.

\section{References}

[1] MoorJ, J. H., Phys. Stat. Sol. (a) 17 (1973) 521.

[2] MooIJ, J. H. and DE JoNG, M., J. Vacuum Sci. Technol. 9 (1972) 446.

[3] Hougton, R. W., Sarachik, M. P. and Kouvel, J. S., Phys. Rev. Lett. 25 (1970) 238.

[4] Hirayama, H., Tanaka, M. and Sakuma, E., Bull. Electrotech. Lab. (Japan) 34 (1970) 56.

[5] SavitskiI, E. M. and Pravoverov, N. L., Sov. Phys., J. Inorg. Chem. 6 (1961) 253.

[6] Brotzen, F. R., Harmon, E. L. Jr and Troiano, A. R., $J$. Metals 7 (1955) 413.

[7] Ahmad, H. M. and Greig, D., Phys. Rev. Lett. 32 (1974) 833.

[8] Mott, N. F., Proc. R. Soc. A 156 (1936) 368.

[9] Coles, B. R. and Taylor, J. C., Proc. R. Soc. A 267 (1962) 139.

[10] Das, S. G., Koelling, D. D. and Mueller, F. M., Solid State Commun. 12 (1973) 89.

[11] Hufner, S., Wertheim, G. K., Cohen, R. L. and WerNICH, J. H., Phys. Rev. Lett. 28 (1972) 488.

[12] NemoshKalenko, V. V., Senkevich, A. I., Mindlina, M. A. and Aleshin, V. G., Phys. Stat. Sol. (b) 56 (1973) 771.

[13] Schwartz, L., Brouers, F., Vedyayev, A. V. and EhrenREICH, H., Phys. Rev. B 4 (1971) 3383.
[14] Chen, A. B., Weisz, G. and Sher, A., Phys. Rev. B 5 (1972) 2897.

[15] Levin, K. and Ehrenreich, H., Phys. Rev. B 3 (1971) 4172.

[16] Brouers, F. and Vedyayev, A. V., Phys. Rev. B 5 (1972) 348.

[17] Brouers, F. and Vedyayev, A. V., in Band Structure Spectroscopy of Metals and Alloys, edited by D. J. Fabian and L. M. Watson (Academic Press, London) 1973.

[18] Gelatt, C. D. Jr. and Ehrenreich, H., Phys. Rev. 10B (1974) 398.

[19] Brouers, F. and Van Der Rest, J., J. Phys. F. : Metal Phys. 2 (1972) 1070

[20] Velicky, B., Kirkpatrick, S. and Ehrenreich, H., Phys. Rev. 175 (1968) 747.

[21] Brouers, F. and Vedyayev, A. V., J. Phys. F. : Metal Phys. 3 (1973) 127.

[22] Velicky, B., Phys. Rev. 184 (1969) 614.

[23] Brouers, F., Vedyayev, A. V. and Giorgino, M., Phys. Rev. B 7 (1973) 380.

[24] Brouers, F., Brauwers, M. and Rivory, J., J. Phys. F. : Metal Phys. 4 (1974) 928.

[25] BrauWERS, M. and Brouers, F., in preparation.

[26] Levin, K. and Mills, D. L., Phys. Rev. B 9 (1974) 2354. 